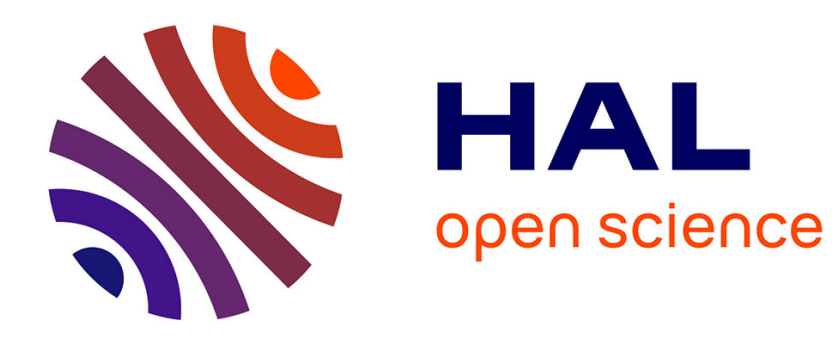

\title{
Inner-Cheeger Opening and Applications
}

Santiago Velasco-Forero

\section{To cite this version:}

Santiago Velasco-Forero. Inner-Cheeger Opening and Applications. Mathematical Morphology and Its Applications to Signal and Image Processing, May 2015, Reykjavik, Iceland. 10.1007/978-3-31918720-4_7 . hal-01250280

\section{HAL Id: hal-01250280 \\ https://hal.science/hal-01250280}

Submitted on 4 Jan 2016

HAL is a multi-disciplinary open access archive for the deposit and dissemination of scientific research documents, whether they are published or not. The documents may come from teaching and research institutions in France or abroad, or from public or private research centers.
L'archive ouverte pluridisciplinaire HAL, est destinée au dépôt et à la diffusion de documents scientifiques de niveau recherche, publiés ou non, émanant des établissements d'enseignement et de recherche français ou étrangers, des laboratoires publics ou privés. 


\title{
Inner-Cheeger Opening and Applications
}

\author{
Santiago Velasco-Forero \\ Centre of Mathematical Morphology \\ Mines Paris Tech - France
}

\begin{abstract}
The aim of this paper is to study an optimal opening in the sense of minimize the relationship perimeter over area. We analyze theoretical properties of this opening by means of classical results from variational calculus. Firstly, we explore the optimal radius as attribute in morphological attribute filtering for grey scale images. Secondly, an application of this optimal opening that yields a decomposition into meaningful parts in the case of binary image is explored. We provide different examples of 2D, 3D images and mesh-points datasets.
\end{abstract}

\section{Introduction}

Many of the methods in mathematical morphology study the relationship between size/shape of objects via max-plus convolution by structuring elements (SE) 1]. One can realize that openings and closings with square, disk or hexagon SEs, are often good enough for some filtering tasks. However, if the structuring elements are able to adapt their shapes and sizes to the image content, some enhancement properties are improved [2,3]. This intuition leads to propose area openings [4, and more generally, to introduce attributes openings [5]. Recently many other attribute filters have been proposed to more specific problems 678 .

In this paper, we introduce a new attribute named "Inner-Cheeger opening" which is, for a binary shape $\mathbf{S}$, the size $t$, such that an opening by a disk of $t$ minimize the relationship perimeter over area: $t$ is named the Cheeger constant of $\mathbf{S}$ in the theory of Variational Calculus 9. Additionally, we explore the application of this operator in the context of decomposition for two-dimensional (2D) and three dimensional (3D) shapes into meaningful parts, which is a challenging problem in image processing [10], pattern recognition [1112, remote sensing [13, and computer vision [14. Thus, we propose a new shape decomposition method, called Inner-Cheeger shape decomposition, denoted by ICSD. This method is characterized by a sequence of Inner-Cheeger openings. Finally, the experimental section includes some examples of 2D, 3D images and mesh-points datasets to illustrate the interest of our method.

\section{Inner-Cheeger opening}

The theory of sets of finite perimeter provides a particularly well-suited framework for studying the existence, symmetry, regularity, and structure of minimizers in those geometric variational problems in which surface area is minimized 
under a volume constraint [9]. In this paper, we consider the follow variational problem and its application to image processing and shape characterization.

\subsection{Formulation}

Given a domain $\mathbf{S}$ of $\mathbb{R}^{d}$, with $d \geq 2$, one is asked to find the Cheeger constant of $\mathbf{S}$, defined as:

$$
h(\mathbf{S}):=\min _{\mathbf{X} \subset \mathbf{S}} \frac{\operatorname{Per}(\mathbf{X})}{\operatorname{Area}(\mathbf{X})},
$$

where $\operatorname{Area}(\mathbf{X})$ is the $(d)$-dimensional volume of $\mathbf{X}$ and $\operatorname{Per}(\mathbf{X})$ is the perimeter of $\mathbf{X}$ or $(d-1)$-dimensional measure. It is important to note that the minimum in (1) is taken over all nonempty sets of finite perimeter contained in $\mathbf{S}$. Thus, any minimiser of (1) is named Cheeger set of $\mathbf{S}$ and it is denoted by $\mathbf{C h}(\mathbf{S})$. Despite this simple formulation of (1), many no trivial questions arise, for instance, the computation, and estimation of $h(\mathbf{S})$ and the characterization of Cheeger sets of $\mathbf{S}$. They have been the main subject of many recent research papers [15]16].

The following are some of the basic properties which are well-known about the Cheeger problem in (1), the proofs of them can be found for instance in 15]16 17/18.

Proposition 1. Let $\mathbf{S}_{1}, \mathbf{S}_{2} \subseteq \mathbb{R}^{d}$ be bounded, open sets. Then the following properties hold.

1. (Existence) There exists a (possibly non-unique) Cheeger set $\mathbf{X} \subseteq \mathbf{S}$.

2. (Decreasing) If $\mathbf{S}_{1} \subset \mathbf{S}_{2}$ then $h\left(\mathbf{S}_{1}\right) \geq h\left(\mathbf{S}_{2}\right)$

3. (Isometry) For any $\lambda>0$ and any isometry $T: \mathbb{R}^{d} \rightarrow \mathbb{R}^{d}$, one has $h(T(\mathbf{S}))=$ $\frac{1}{\lambda} h(\mathbf{S})$

4. (Intersection with the boundary) The minimum in (1) is attained at a subset $\mathbf{X} \subseteq \mathbf{S}$ such that $\partial \mathbf{X}$ intersect $\partial \mathbf{S}$.

5. (Closure with union and intersection) If $\mathbf{X}_{1}$ and $\mathbf{X}_{2}$ are Cheeger in $\mathbf{S}$, then $\mathbf{X}_{1} \cup \mathbf{X}_{2}$ and $\mathbf{X}_{1} \cap \mathbf{X}_{2}$ (if it is not empty) are also Cheeger in $\mathbf{S}$.

Many other properties can be found in $16[18$. However, we have included only the most relevant for our work.

\subsection{Convex case}

In the case of $\mathbf{S}$ is convex, we can add to the list of properties in Proposition 1. the uniqueness and convexity of Cheeger sets, which has been proved in [16]. However, finding the Cheeger set of a given $\mathbf{S}$ is a difficult task. This task is simplified if $\mathbf{S}$ is a convex set and $d=2$. In this situation is follows from [17, Theorem 3.32 that Cheeger $=\mathbf{C h}(\mathbf{S})$ is convex and uniquely defined as the union of a set of disks of suitable radius [15. Thus, the Cheeger set in $\mathbf{S}$ is unique and is identified by the following theorem. 
Theorem 1. 1519] Let $\mathbf{S} \subseteq \mathbb{R}^{2}$ be a nonempty bounded convex set. There exist a unique value $t>0$ such that $\operatorname{Area}\left(\mathbf{S}^{t}\right)=\pi t^{2}$. Then $h(\mathbf{S})=\frac{1}{t}$ and the $\boldsymbol{C h}(\mathbf{S})=\delta_{B(t)}\left(\mathbf{S}^{t}\right)$, where $B(t)$ denotes a ball of radius $t$ and $\mathbf{S}^{t}=\{x \in \mathbf{S}$ : $\operatorname{dist}(x, \partial \mathbf{S})>t\}$ is called the inner Cheeger set of $\mathbf{S}$.

The proof of Theorem 1 is basically based on the Steiner's formulae in the case of $d=2[5]$ and it is included in Appendix 1 .

Corollary 1. For a nonempty bounded convex set $\mathbf{S} \in \mathbb{R}^{2}$, the Cheegger set $\boldsymbol{C h}(\mathbf{S})$ is an opening with a disk of radius given by the Theorem 1 as structuring element.

In the sequel, we use the name "Inner-Cheeger opening" to the opening defined in Corollary 1.

\subsection{Small perturbations}

Additionally, it is important to note that the uniqueness of Cheeger sets holds up to arbitrary small perturbations of $\mathbf{S}[20$.

Theorem 2. 20] Let $\mathbf{S} \subseteq \mathbb{R}^{N}$ be an open set with finite volume. Then for any compact set $K \in \mathbf{S}$, there exist a bounded open set $\mathbf{S}_{K} \subset \mathbf{S}$ such that $K \subset \mathbf{S}_{K}$ and $\mathbf{S}_{K}$ has a unique Cheeger set.

A visual example of this property is illustrated in Fig. 1

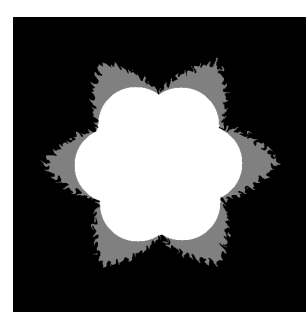

(a) $t=65$

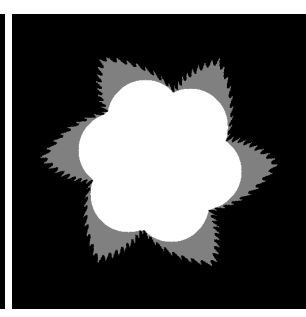

(b) $t=65$

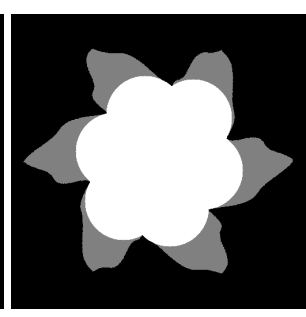

(c) $t=67$

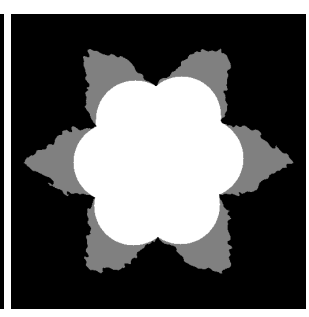

(d) $t=67$

Fig. 1. Example of invariance of Inner-Cheeger opening to small perturbations of the shape. (b) and (d) are perturbations of (a) and (c) respectively.

\subsection{Comparison with skeleton transformation}

The skeleton transformation is a widely used transformation in the field of image processing. The definition of skeleton was introduced by [21, proposing the grass fire analysis: the skeleton consists of the points where different fire fronts intersect, or quench points. Later, another formal definition of the skeleton was 
proposed by Calibi in 1965 22] which relies on the concept of maximal ball. The skeleton is defined as the set of the maximal balls. In [22, it was proved that the notion of quench points and centers of maximal balls are equivalent. According to Chapter one of [1, the notion of maximal ball has been known since the 30s 23124. A skeleton transformation has to obey the following properties to make its results convenient for the global representation of objects 25: Homotopy: the skeleton transform must preserve the topology of the initial object; Rotation invariance: the skeleton transform of a rotated shape should be the rotated of the skeleton; Reconstruction: the initial shape should be reconstructed from the skeleton transform. For continuous images, the skeleton $s k(\mathbf{S})$ of a shape $\mathbf{S}$ is given by the Lantuéjoul's formulate 2627. defined as follows:

$$
s k(\mathbf{S})=\bigcup_{\rho>0} \bigcap_{\mu>0}\left[\varepsilon_{\rho B}(\mathbf{S})-\left(\gamma_{B}\left(\varepsilon_{\rho B}(\mathbf{S})\right)\right)\right]
$$

A ball $B$ included in $\mathbf{S}$ is said to be maximal if and only if there exist no other ball included in $\mathbf{S}$ and containing $B, \forall B^{\prime}$ ball, $B \subseteq B^{\prime} \subseteq \mathbf{S} \Rightarrow B^{\prime}=B$,i.e., a ball is maximal in the shape if it is not included in any single other ball in the shape. We denote $M B T(\mathbf{S})=\{x \in \mathbf{S} ; \exists$ a maximal ball centred at $x\}$, the set of all the centres of the maximal balls included in $\mathbf{S}$, and $r_{x}$ the corresponding radius of it associated maximal ball. The skeleton $s k(\mathbf{S})$ of a set $\mathbf{S}$ is then defined as the set of the centers of its maximal balls:

$$
s k(\mathbf{S})=\left\{x \in \mathbf{S}, \exists r_{x} \geq 0, B\left(x, r_{x}\right) \text { maximal ball of } \mathbf{S}\right\} .
$$

This collection of maximal balls $M B T(\mathbf{S})$ is equivalent to the shape $\mathbf{S}$ itself since one has the reconstruction formula:

$$
\mathbf{S}=\bigcup_{x \in M B T(\mathbf{S})} \delta_{r_{x}}(x)
$$

In this representation, we can easily see that for $\mathbf{S} \subseteq \mathbb{R}^{2}$ the Inner-Cheeger opening is

$$
\mathbf{C h}(\mathbf{S})=\bigcup_{x \in M B T(\mathbf{S}), r_{x} \geq t} \delta_{r_{x}}(x),
$$

where $t$ is defined by Theorem 1 . An example of the collection of maximal balls and the correspondent Inner-Cheeger set is shown in Fig. 2.

\section{Applications}

In this section we present some examples to illustrate our methods. We present two applications: Attribute filtering and shape decomposition in $2 \mathrm{D}, 3 \mathrm{D}$ and mesh shapes. 


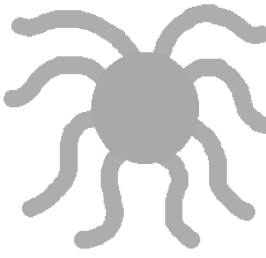

(a) $\mathrm{S}$

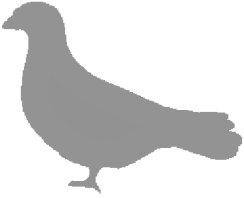

(e) $\mathbf{S}$

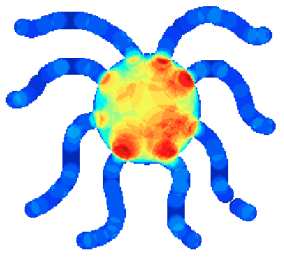

(b) Reconstruction from the $M B T(\mathbf{S})$

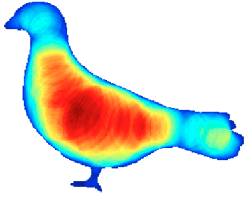

(f) Reconstruction from the $M B T(\mathbf{S})$

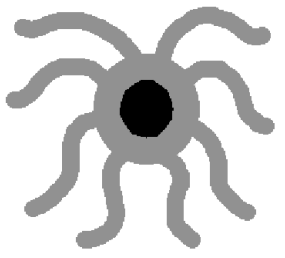

(c) $\operatorname{InnerCh}(\mathbf{S})$

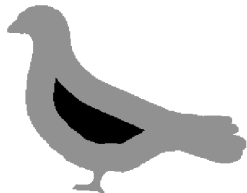

(g) InnerCh(S)

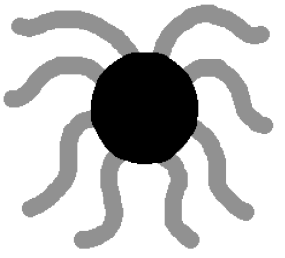

(d) $\mathrm{Ch}(\mathrm{S})$

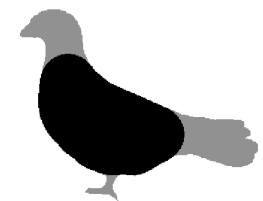

(h) $\mathbf{C h}(\mathbf{S})$

Fig. 2. Comparison of Inner-Cheeger opening and Skeleton transformation for two images. Colours in (b) and (f) show of the number of balls in the reconstruction of MBT.

\subsection{Inner-Cheeger set as an attribute filter}

Many works about attribute filters have been presented in the literature [28]. Most of them address the problem of filter-out target objects and preserving the contours of non-interesting objects. In this first application, we start with the description of a grey scale image $\mathbf{I}$ by its upper level set decomposition:

$$
\mathbf{I}(x)=\sup (i: \mathbf{I}(x) \geq i \text { is true })
$$

where is discrete images $i=\left\{0,1, \ldots, t_{\max }\right\}$ and $t_{\max }$ stands by the maximum grey scale value of the image. Now, following [5], we can define an attribute filter on $\mathbf{I}$ by the well-known threshold decomposition principle:

$$
\psi(\mathbf{I})(x)=\sup (i: \Psi(\mathbf{I}(x) \geq i) \text { is true })
$$

where $\Psi$ is a binary attribute filter. The advantage of this representation is the fact that most of the properties that are known to hold for $\Psi$ are also true for its grey-level counterpart $\psi$. Mainly, if $\Psi$ is an increasing criterion, it was proved if [5] that $\psi$ is a valid opening in the morphological sense. We propose to use the optimal value of $t$ is Theorem 1 as attribute to describe connected components in the threshold decomposition in Eq. 7. In proposition 1, property 2 , we have stated that our attribute is increasing, which allows the use a fast implementation (by reducing the search space in Theorem 11), for instance, by 
means of a max-tree structure 29]. Some example of this attribute filter are illustrated in Fig. 3 for different values of $t$. This attribute opening preserves contours of object with largest Cheeger set, i.e., objects with large sphericity.

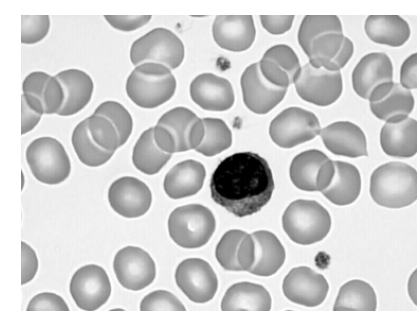

(a) Original Image

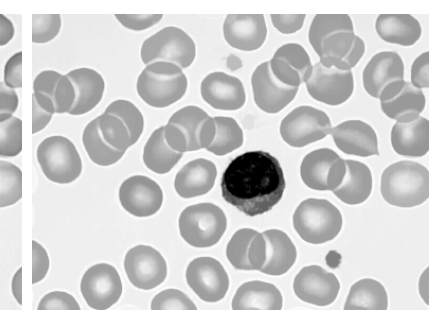

(b) Opening by Cheeger Atribute $(t=4)$

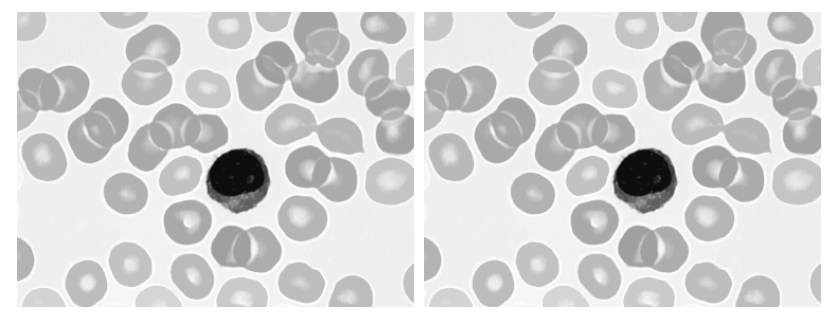

(c) Opening by Cheeger (d) Opening by Cheeger Atribute $(t=6) \quad$ Atribute $(t=8)$

Fig. 3. Inner-Cheeger opening for different values of proposed attribute.

\subsection{Shape Decomposition}

Instead of characterizing an object as a whole, part-based representation deals with a representation where an object is decompose it into a number of "natural parts [30]. The aim of this shape decomposition is to simplify a given shape into meaningful parts to make easier its analysis. Many morphological-based operators approaches 3113233 have been formulated with the intuition of finding the "best" opening (with a ball as structuring element) by means of an index considering the size "part/shape" ratio and "part/convex hull" ratio. However, these methods are often hard to implement and/or do require the solution of difficult optimization problem. In this section we propose to use the inner Cheeger decomposition to perform shape decomposition. For a given shape $\mathbf{S}$, we firstly compute the $\mathbf{C h}(\mathbf{S})$ and successively the same transformation is applied to each connected component of the difference between $\mathbf{S}$ and $\mathbf{C h}(\mathbf{S})$. We note that many of the connected components can have small areas, so a threshold parameter is 
included to obtain only meaningful parts of the object. We use the term InnerCheeger shape decomposition (ICSD) to refer this sequential separation, due to the fact that in the case of non-convex shapes, one cannot say that this procedure find a valid Cheeger set. However, as it is illustrated in Fig, 4, the decomposition determines relevant parts and as in most of the distance based transformation, the result is robust to articulate transformations. In addition, in our case it is robust to small perturbations of the original shape. At this point, we can use a

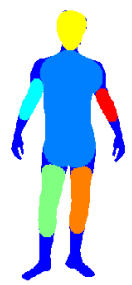

(a)

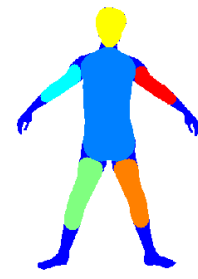

(b)

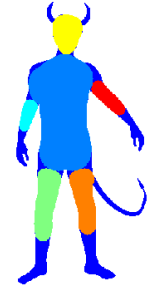

(c)

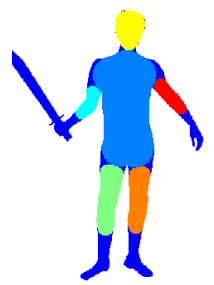

(d)

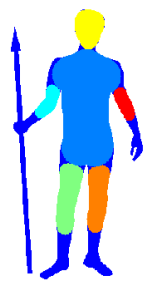

(e)

Fig. 4. Example of decomposition by Iterative Cheeger Openings (2 Levels). Colour are associated with the order of discovery in the iteration of ICSD. Note that results are robust to modification in the human shape.

similar approach to 3D shapes in Fig. 5 and 3D points clouds in Fig. 6. However, the optimal value of $t$ in Theorem 1 cannot be applied directly for $3 \mathrm{D}$ shapes. Thus, assuming that the inner set of a convex 3D shape if composed by the union of balls, we can obtain an equivalent expression for three dimensional objects by using the Steiner's formula as it is shown in Appendix. Accordingly, in 3D shape, we should look for the radius $t$ that $\operatorname{Vol}\left(\mathbf{S}^{t}\right)+\operatorname{MeanB}\left(\mathbf{S}^{t}\right) t^{2}\left[\frac{1}{2 \pi}-1\right]=\frac{8 \pi r^{3}}{3}$, where $\operatorname{MeanB}(\mathbf{S})$ stands for mean breadth of the binary shape $\mathbf{S}$. A Fig. 5 illustrates the results of ICSD for three postures for the same 3D human model. Additionally, we explore the application of our approach in mesh of points in three different frames of "Ben Walking Scene"

\section{Conclusion}

We have defined an optimal opening via Inner-Cheeger sets. We have explored some important morphological properties including the robustness against small perturbations. Finally, we have illustrated the interest and behavior of such operators in some problems of image processing and shape decomposition. Future work includes the analysis of similar problem in shapes with holes as it is the case of [34] and compare our results with the space of shapes from [35].

\footnotetext{
1 http://4drepository.inrialpes.fr/pages/home
} 


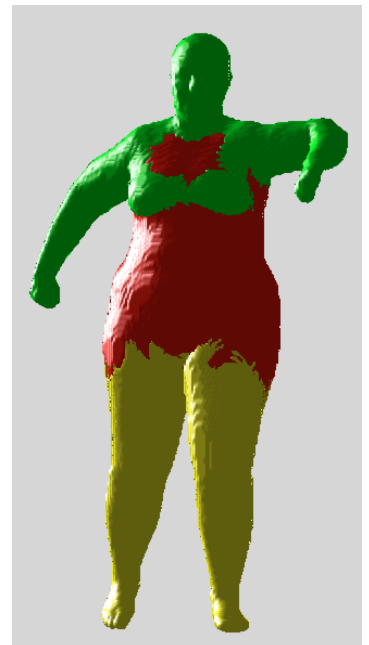

(a)

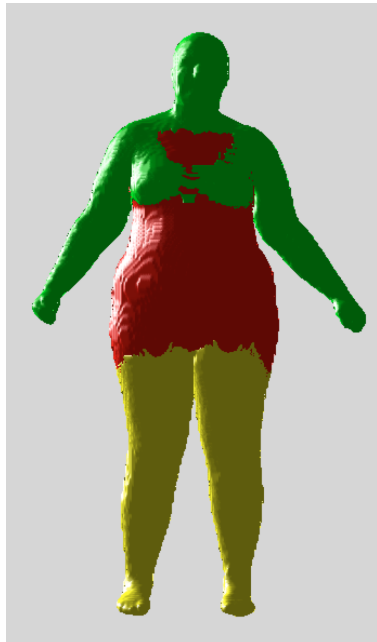

(b)

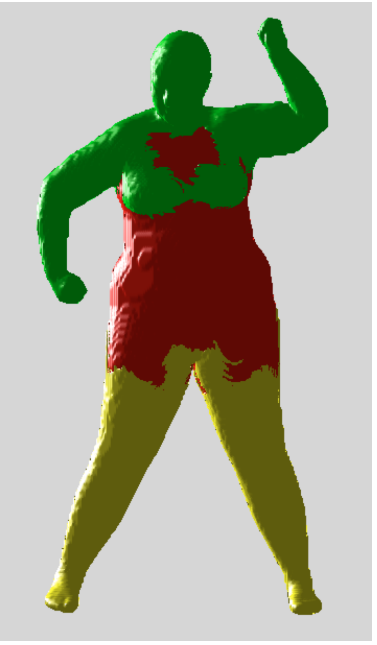

(c)

Fig. 5. Three examples of proposed decomposition for 3D shapes. Colour are associated with the order of discovery in the iteration of ICSD. Note that results are robust to changes in the pose of the human shape.

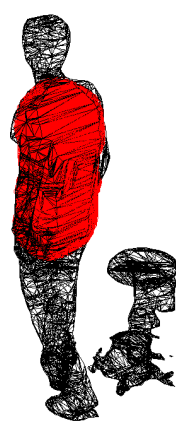

(a) Frame 103

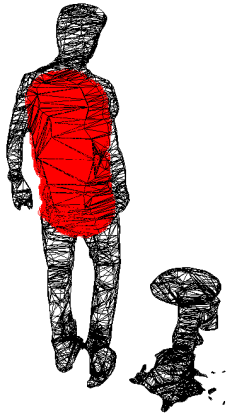

(b) Frame 118

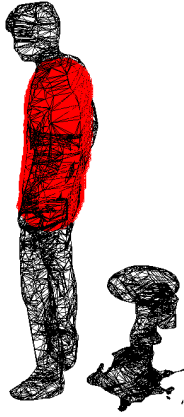

(c) Frame 134

Fig. 6. In red the points identified as part of the Cheeger opening for meshes (black lines) in some frames of "Ben Walking" 4D dataset. The inner-Cheeger set in this case is the thorax of "Ben". The results are quite robust considering that the input data includes the presence of the "chair" and different postures of "Ben" 


\section{Appendix}

We follow the results from [15]19] for 2D shapes to obtain:

$$
\begin{array}{r}
h(\mathbf{S})=\frac{1}{r}=\frac{\operatorname{Per}\left(\delta_{r B}(\mathbf{S})\right)}{\operatorname{Area}\left(\delta_{r B}(\mathbf{S})\right)}=\frac{\operatorname{Per}(\mathbf{S})+2 \pi r}{\operatorname{Area}(\mathbf{S})+\operatorname{Per}(\mathbf{S}) r+\pi r^{2}} \\
\operatorname{Area}(\mathbf{S})+\operatorname{Per}(\mathbf{S}) r+\pi r^{2}=\operatorname{Per}(\mathbf{S}) r+2 \pi r^{2} \\
\operatorname{Area}(\mathbf{S})=\pi r^{2}
\end{array}
$$

In the case of $d=3$, the Steiner's formula gives explicitly for a compact convex:

$$
\begin{array}{r}
\operatorname{Vol}\left(\delta_{r B}(\mathbf{S})\right)=\operatorname{Vol}(\mathbf{S})+\operatorname{Sur}(\mathbf{S}) r+\operatorname{MeanCurv}(\mathbf{S}) r^{2}+\frac{4 \pi}{3} r^{3} \\
\operatorname{Sur}\left(\delta_{r B}(\mathbf{S})\right)=\operatorname{Sur}(\mathbf{S})+2 \pi \operatorname{MeanCurv}(\mathbf{S}) r+4 \pi r^{2} \\
\operatorname{MeanCurv}\left(\delta_{r B}(\mathbf{S})\right)=\operatorname{MeanCurv}(\mathbf{S})+4 \pi r
\end{array}
$$

Additionally, it is important to note that the mean breadth, denoted by $\operatorname{MeanB}(\mathbf{I})$, connects the integral of the mean curvature MeanCurv(S) for an object $\mathbf{I}$ whose boundary is of class $\mathbb{C}^{2}$ by the formula, $\operatorname{MeanB}(\mathbf{I})=2 \pi \operatorname{MeanCurv}(\mathbf{S})$.

$$
h(\mathbf{S})=\frac{1}{r}=\frac{\operatorname{Sur}\left(\delta_{r B}(\mathbf{S})\right)}{\operatorname{Vol}\left(\delta_{r B}(\mathbf{S})\right)}=\frac{\operatorname{Sur}(\mathbf{S})+2 \pi \operatorname{MeanCurv}(\mathbf{S}) r+4 \pi r^{2}}{\operatorname{Vol}(\mathbf{S})+\operatorname{Sur}(\mathbf{S}) r+\operatorname{MeanCurv}(\mathbf{S}) r^{2}+\frac{4 \pi r^{3}}{3}}
$$

and after simplification, we obtain $\frac{8 \pi r^{3}}{3}=\operatorname{Vol}(\mathbf{S})+\operatorname{MeanB}(\mathbf{S}) r^{2}\left[\frac{1}{2 \pi}-1\right]$. We remark that the percolation analysis in three-dimensional objects in [36] uses similar results in random models with spheres and Poisson polyhedra as primary grains.

\section{References}

1. L. Najman and H. Talbot, Mathematical morphology: from theory to applications. ISTE-Wiley, Jun. 2010.

2. J. Angulo and S. Velasco-Forero, "Structurally adaptive mathematical morphology based on nonlinear scale-space decompositions," Image Analysis $\&$ Stereology, vol. 30, no. 2, pp. 111-122, 2011.

3. V. Ćurić, A. Landström, M. J. Thurley, and C. L. Luengo Hendriks, "Adaptive mathematical morphology-a survey of the field," Pattern Recognition Letters, vol. 47 , pp. 18-28, Oct. 2014.

4. L. Vincent, "Grayscale area openings and closings, their efficient implementation and applications," in Proceedings of the Conference on Mathematical Morphology and its Applications to Signal Processing, may 1993, pp. 22-27.

5. E. J. Breen and R. Jones, "Attribute openings, thinnings, and granulometries," Computer Vision and Image Understanding, vol. 64, no. 3, pp. 377-389, 1996.

6. H. Talbot and B. Appleton, "Efficient complete and incomplete path openings and closings," Image Vision Comput., vol. 25, no. 4, pp. 416-425, Apr. 2007. 
7. A. Serna and B. Marcotegui, "Attribute controlled reconstruction and adaptive mathematical morphology," in Mathematical Morphology and Its Applications to Signal and Image Processing. Springer, 2013, pp. 207-218.

8. V. Morard, E. Decencière, and P. Dokládal, "Efficient geodesic attribute thinnings based on the barycentric diameter," Journal of mathematical imaging and vision, vol. 46, no. 1, pp. 128-142, 2013.

9. F. Maggi, Set of Finite Perimeter and Geometric Variational Problems. Cambridge University Press, 2012.

10. B. M. Mehtre, M. S. Kankanhalli, and W. F. Lee, "Shape measures for content based image retrieval: a comparison," Information Processing \& Management, vol. 33, no. 3, pp. 319-337, 1997.

11. A. Cerri, S. Biasotti, M. Abdelrahman, J. Angulo, K. Berger, L. Chevallier, M. ElMelegy, A. Farag, F. Lefebvre, A. Giachetti et al., "Shrec'13 track: retrieval on textured 3d models," in Proceedings of the Sixth Eurographics Workshop on 3D Object Retrieval. Eurographics Association, 2013, pp. 73-80.

12. S. Velasco-Forero and J. Angulo, "Statistical shape modeling using morphological representations," in Pattern Recognition (ICPR), 2010 20th International Conference on. IEEE, 2010, pp. 3537-3540.

13. L. Gueguen, "Classifying compound structures in satellite images: A compressed representation for fast queries," Transactions on Geoscience and Remote Sensing, pp. 1-16, 2014.

14. L. Younes, "Spaces and manifolds of shapes in computer vision: An overview," Image and Vision Computing, vol. 30, no. 6, pp. 389-397, 2012.

15. B. Kawohl and T. Lachand-Robert, "Characterization of Cheeger sets for convex subsets of the plane," Pac. J. Math., vol. 225, no. 1, pp. 103-118, 2006.

16. F. Alter and V. Caselles, "Uniqueness of the cheeger set of a convex body," Nonlinear Analysis, vol. 70, no. 1, pp. 32-44, 2009.

17. E. Stredulinsky and W. Ziemer, "Area minimizing sets subject to a volume constraint in a convex set," The Journal of Geometric Analysis, vol. 7, no. 4, pp. 653-677, 1997. [Online]. Available: http://dx.doi.org/10.1007/BF02921639

18. G. P. Leonardi and A. Pratelli, "On the cheeger sets in strips and non-convex domains," arXiv preprint arXiv:1409.1376, 2014.

19. V. C. F. Alter and A. Chambolle, "Evolution of characteristic functions of convex sets in the plane by the minimizing total variation flow," Interfaces and Free Boundaries, vol. 7, 2005.

20. V. Caselles, A. Chambolle, and M. Novaga, "Some remarks on uniqueness and regularity of cheeger sets," Rend. Semin. Math. Univ. Padova, vol. 123, p. 191201, 2010.

21. H. Blum, "A transformation for extracting new descriptors of shape," in Proceedings of a Symposium on Models for the Perception of Speech and Visual Forms. Boston: MIT, Nov 1967.

22. L. Calabi, A study of the skeleton of plane figures. Parke Mathematical Laboratories, 1965.

23. G. Durand, "Théprie des ensembles. points ordinaires et point singuliers des enveloppes de sphères," Comptes-rendus de l'Acad'emie de Sciences, vol. 190, pp. 571-573, 1930.

24. G. Bouligand, Introduction à la gémétrie infinitésimale directe. Vuibert, 1932.

25. G. Malandain and S. Fernández-Vidal, "Euclidean skeletons," Image and vision computing, vol. 16, no. 5, pp. 317-327, 1998.

26. C. Lantuejoul, "La squelettisation et son application aux mesures topologiques des mosaiques polycristallines," Ph.D. dissertation, Ecole des Mines de Paris, 1978. 
27. — - "Skeletonization in quantitative metallography," Issues of Digital Image Processing, vol. 34, no. 107-135, p. 109, 1980.

28. P. Salembier and J. Serra, "Flat zones filtering, connected operators, and filters by reconstruction," Image Processing, IEEE transactions on, vol. 4, no. 8, pp. 1153-1160, 1995.

29. E. Carlinet and T. Géraud, "A comparison of many max-tree computation algorithms," in Mathematical Morphology and Its Applications to Signal and Image Processing. Springer, 2013, pp. 73-85.

30. D. D. Hoffman and W. A. Richards, "Parts of recognition," Cognition, vol. 18, no. 1, pp. 65-96, 1984.

31. J. Xu, "Morphological decomposition of 2-d binary shapes into convex polygons: A heuristic algorithm," Image Processing, IEEE Transactions on, vol. 10, no. 1, pp. 61-71, 2001.

32. L. Yu and R. Wang, "Shape representation based on mathematical morphology," Pattern Recognition Letters, vol. 26, no. 9, pp. 1354-1362, 2005.

33. D. H. Kim, I. D. Yun, and S. U. Lee, "A new shape decomposition scheme for graph-based representation," Pattern Recognition, vol. 38, no. 5, pp. 673-689, 2005.

34. G. Liu, Z. Xi, and J.-M. Lien, "Dual-space decomposition of 2 d complex shapes," in 27th IEEE Conference on Computer Vision and Pattern Recognition (CVPR). Columbus, OH: IEEE, Jun. 2014.

35. Y. Xu, T. Géraud, and L. Najman, "Morphological filtering in shape spaces: Applications using tree-based image representations," in Pattern Recognition (ICPR), 2012 21st International Conference on. IEEE, 2012, pp. 485-488.

36. D. Jeulin, "Random structures in physics," in Space, Structure and Randomness, Contributions in Honor of Georges Matheron in the Fields of Geostatistics, Random Sets, and Mathematical Morphology, ser. Lecture Notes in Computer Science. Springer Berlin / Heidelberg, 2005, vol. 183, pp. 183-222. 